Cahiers « Mondes anciens »

MONDES

ANCIENS

Histoire et anthropologie des mondes anciens

$6 \mid 2015$

Mères grecques

\title{
Les relations mère-enfant dans le corpus homérique
}

The Relations Mother-Son in the Homeric Corpus

\section{Susana Reboreda Morillo}

\section{(2) OpenEdition}

Journals

Édition électronique

URL : http://journals.openedition.org/mondesanciens/1288

DOI : 10.4000/mondesanciens. 1288

ISSN : 2107-0199

Éditeur

UMR 8210 Anthropologie et Histoire des Mondes Antiques

Référence électronique

Susana Reboreda Morillo, «Les relations mère-enfant dans le corpus homérique », Cahiers "Mondes anciens » [En ligne], 6 | 2015, mis en ligne le 27 janvier 2015, consulté le 19 avril 2019. URL : http:// journals.openedition.org/mondesanciens/1288; DOI : 10.4000/mondesanciens.1288

Ce document a été généré automatiquement le 19 avril 2019

Licence Creative Commons

Les Cahiers «Mondes Anciens » sont mis à disposition selon les termes de la licence Creative

Commons Attribution - Pas d'Utilisation Commerciale - Pas de Modification 4.0 International. 


\section{Les relations mère-enfant dans le corpus homérique}

The Relations Mother-Son in the Homeric Corpus

Susana Reboreda Morillo

\section{Introduction}

1 Bien qu'une grande partie de ma trajectoire de recherche se limite aux poèmes homériques, je dois admettre que chaque fois que je me centre sur un nouveau thème, la multitude de nouvelles informations obtenues de l'analyse de ces vers m'étonne. Des vers qui, de plus, se sont imposés comme modèle dans de nombreux domaines de la vie des Grecs dans l'Antiquité1.

2 Dans le cas concret qui nous occupe, l'analyse de la maternité chez Homère, les difficultés qui surgissent à première vue sont considérables. La première est issue de la thématique principale des deux poèmes : la colère d'Achille envers Agamemnon et ses conséquences au cours de la guerre de Troie dans l'Iliade, et le nostos d'Ulysse, c'est-à-dire les aventures et les difficultés du dernier héros à rentrer chez lui à la fin de la guerre de Troie. Au-delà $\mathrm{du}$ fait que les protagonistes sont principalement des hommes, leurs exploits s'accomplissent, plus dans l'Iliade que dans l'odyssée, dans des contextes très masculins.

3 À cette réalité s'ajoute le fait que la maternité n'était pas un thème central pour un auteur de l'Antiquité; qu'aucune sensibilité spécifique en rapport avec cette réalité n'apparaisse dans les poèmes homériques n'a donc rien d'étrange. Malgré tout, il est possible de mettre en évidence la grandeur et l'importance de la maternité parce que, d'une certaine façon, elle «se faufilait " probablement de manière inconsciente dans les endroits les plus inattendus. Ce sont ces « incursions », involontaires, reflétées chez des personnages ou des situations qui sont au second plan, que nous nous proposons d'analyser dans le contexte des poèmes homériques afin d'en extraire certaines informations. 
Ce que je propose c'est de recueillir ces indices de la relation mère/fils/fille en suivant, dans la mesure du possible, l'ordre des phases de la croissance et du développement de l'enfant depuis la plus tendre enfance jusqu'à la maturité ; choix qui m'obligera à réaliser des sauts d'une œuvre à l'autre. Les analyses incluront aussi bien des références aux humains qu'au monde divin ; cette liberté est admissible dans l'univers religieux grec du fait des abondantes interférences entre les deux contextes, divin et terrestre. De plus, dans certains exemples, comme le cas des déesses qui sont mères de fils mortels, il est possible d'extrapoler certains sentiments et situations (De Paco Serrano 2007). Comme l'affirme Laura Slatkin (2011, p. 24), l'Iliade fait référence explicitement à Thétis dans ses attributs de mère éducatrice et protectrice.

5 La « question homérique » continue à être une grande énigme pour les spécialistes et les opinions sont très diverses. Personnellement, ma position est la suivante: les textes homériques laissent entrevoir certaines caractéristiques de différentes périodes historiques, depuis le monde mycénien jusqu'à la période archaïque ; l'essentiel se situe entre les $\mathrm{IX}^{\mathrm{e}}$ et $\mathrm{VIII}^{\mathrm{e}} \mathrm{s}$. av.J.-C., et se rapporte à certains aspects des formes de vie aristocratique antérieures à l'établissement du système de l'époque classique des poleis.

\section{Être femme chez Homère : épouse et mère}

6 L'activité principale des « aristocrates homériques » consistait à faire la guerre, comprise comme une façon légitime de s'enrichir ; cette exclusivité, reflétée dans la lutte au corps à corps, justifiait le monopole de la participation à la vie politique, l'autre occupation de la classe dirigeante.

7 Quant à leurs femmes, à travers ces deux œuvres, il est possible de pressentir que la société manifestait envers elles bien plus de considération, de tendresse et de respect que ce que laisseront entrevoir postérieurement les époques archaïque et classique. Il est aussi évident que celles-ci étaient laissées à l'écart des occupations qui régentaient les aspects de la vie apparemment estimés comme les plus importants (Mossé 1983, p. 17-34). En général, leur nom était lié à celui d'un homme (Scheid-Tissiner 1999, p. 38-89), plus en tant que filles et épouses qu'en qualité de mères; probablement pour signaler de quel homme elles dépendaient. La plus grande déchéance qu'elles pouvaient subir était de devenir un bien précieux intégré dans le butin de guerre des vaincus; c'est-à-dire de devenir esclave et, la plupart du temps, concubine de ceux qui avaient tué des êtres chers et mis à sac leur terre natale.

8 Malgré ces aspects vraiment cruels, la femme de l'aristocratie homérique manifestait une certaine indépendance et une capacité de décision sur son avenir et, sur elle, reposaient en même temps la gestion et la responsabilité de l'oikos (Leduc 2001, p. 276-280), spécialement quand son époux était sur le champ de bataille. Outre ces prérogatives, elles étaient éduquées pour être de parfaites épouses et de bonnes mères, ce dernier facteur étant indispensable pour perpétuer la lignée, maintenir la force des liens de sang et répéter les devoirs de la société. L'idée transmise par les textes est que le trait le plus spécifique de la femme, celui qui la différenciait et l'opposait à l'homme, était précisément la maternité, comprise comme procréation liée à l'allaitement. En ce sens, María Teresa Molinos Tejada (2005, p. 59) indique que la notion incluse dans le mot matêr ne correspondait pas exactement à celle qui l'est dans le terme patêr : dans le premier cas, le terme peut s'appliquer à un animal femelle avec des petits tandis que le second ne 
s'emploie jamais pour les animaux; cette distinction s'explique par le fait que l'on considérait la maternité comme quelque chose de naturel, tandis que la paternité était une réalité sociale et culturelle.

9 En plus de la procréation, la femme de l'oikos, secondée particulièrement par l'esclave qui exerçait les fonctions de nourrice (Molinos Tejada 2001), surveillait l'éducation et la croissance de ses enfants pendant la première étape de leur vie. L'autre activité majeure des femmes, indépendamment de leur classe sociale, reposait sur le tissage. Toutes les femmes, depuis leur plus tendre enfance, étaient instruites dans l'art de tisser, la différence entre les unes et les autres était la qualité de leur ouvrage et qui en serait le destinataire (Bruit Zaidman 2001, p. 413-417).

\section{La première étape de la vie}

Bien que nous n'ayons pas d'informations sur la conception et la grossesse, il existe quelques allusions relatives au moment de l'accouchement, clairement assimilé à une grande douleur (Molas Font 2002, p. 153-178). Il est symptomatique que, pour renforcer une situation de souffrance extrême chez Agamemnon, on fasse appel à la comparaison des douleurs subies par les femmes qui accouchent: «Elles sont semblables au trait lancinant, cruel, qui frappe une femme en travail, le trait décoché par les Ilithyes, les déesses des enfantements douloureux... Aussi lancinantes sont les peines qui pénètrent alors l'Atride... » (Iliade, XI, 269-271)². Même si les divinités associées à l'accouchement dans la Grèce ancienne sont nombreuses, les plus représentatives étaient Ilithye et Artémis. La première, apparentée particulièrement à ces douleurs, est présente à de nombreuses occasions dans les poèmes.

11 Les poèmes homériques soulignent le lien puissant qui s'établit, à partir de la naissance, entre une mère et ses enfants et qui se démontre de façons diverses. La célèbre scène que l'on connaît traditionnellement sous le nom des « adieux d'Hector à Andromaque » (Iliade, VI, 400-500) nous éloigne du feu de la bataille et de la cruauté des combats pour nous faire plonger dans un environnement domestique où la tendresse occupe une place de choix. Les témoignages de tendresse entre les époux ou des parents envers leur enfant sont si évidents que, comme l'affirment Bile et Klein (2008), même les scholiastes manifestent leur gêne. L'amour envers son épouse, qu'Hector exprime si ouvertement, ainsi que les gestes d'affection envers leur fils constituent une indication supplémentaire de cette plus grande considération de la femme dans les poèmes homériques, ce qui sera inconcevable dans les périodes ultérieures. Dans ce passage ces sentiments sont bien privilégiés par rapport à l'amour maternel, axé sur la crainte pour l'avenir de l'enfant si le héros était mort au combat (De Nazaré Ferreira, p. 73 et 75), ou par rapport à l'attitude paternelle, comme il ressort clairement de la rigoureuse analyse de Le Meur-Weissman (2009, p. 33-35).

12 Les femmes de la noblesse partageaient, en proportion plus ou moins grande, à divers degrés, les tâches de l'allaitement et de l'éducation de leurs enfants avec des nourrices. S'il est difficile d'établir des généralités quant aux occupations des unes ou des autres, il est possible de déduire de quelques vers des œuvres que c'était bien les mères qui allaitaient leurs enfants. C'est l'unique façon de comprendre la requête désespérée d'Hécube à son fils dans un moment aussi difficile que celui de l'affrontement avec Achille et qui le conduira à la mort : « Hector, mon enfant, aie du respect de ce sein. Et de moi aie 
pitié, de moi qui t'ai jadis offert cette mamelle où s'oublient les soucis; souviens-t'en mon enfant !...» (Iliade, XXII, 82-86).

Il y a, de plus, des preuves qu'élever les enfants impliquait pour les mères un affaiblissement, réalité qui pourrait être interprétée en relation avec le fait d'allaiter ${ }^{3}$. Ces trois circonstances, accoucher, allaiter et élever apparaissent souvent liées et sont à la base du lien fort et réciproque qui s'établit entre les mères et leurs enfants. Comme nous l'avons vu dans le cas d'Hécube, il était habituel que, face à une situation limite ou face à la pression pour que les mères changent d'attitude, ces dernières évoquent ces sacrifices. En outre, les mères divines, plus que les autres, ne cessent dans l'épopée de vouloir protéger leur progéniture. Pour leur part, les enfants manifestaient à leur mère un grand respect, ce qui n'impliquait pas qu'ils cèdent automatiquement à leurs suppliques. Ainsi, par exemple, Achille adresse le message suivant à Thétis: "Ne cherche pas, quelle que soit ta tendresse, à me tenir loin du combat; aussi bien ne t'écouterai-je pas » (Iliade, XVIII, 125-126).

L'image de la nourrice est incarnée, dans l'Odyssée, par le personnage d'Euryclée, une esclave achetée jeune, éduquée dans l'oikos, et qui devient une personne de confiance. Euryclée avait participé aux premiers soins d'Ulysse puis à ceux de son fils, Télémaque ( Odyssée, I, 427-432).

15 Au-delà de cet aspect, ces mères sans grandes occupations ou options de loisirs, à l'exception des réunions sociales et religieuses avec les autres femmes, devaient probablement voir, à travers leurs enfants, une manière de canaliser l'affection de façon continue ce qui compensait, tout au moins en partie, les longues absences de leurs époux à cause de la guerre. Elles transmettaient aussi à leurs enfants pendant cette première étape de la vie si marquante les bases des valeurs qui permettaient de perpétuer la société, tout en prenant soin d'eux et les protégeant. Il est ainsi révélateur, par exemple, que, pour illustrer le moment où la déesse Athéna dévie une flèche destinée à Ménélas, on fasse appel à l'analogie de la maternité « [...] tout comme une mère éloigne une mouche d'un fils qui s'étend pour un doux sommeil» (Iliade, IV, 130-133). Luísa De Nazarés Ferreira (2000, p. 57-60 et p. 67-68) inclut cette phrase dans un groupe de comparaisons imagées ayant pour thème les liens d'affection et de protection entre les mères et leurs enfants. Le moment où Teucros s'abrite derrière le bouclier d'Ajax pour tirer une flèche de son arc renverrait également à l'image d'un enfant cherchant la protection de sa mère (VIII, 271-272). Finalement, la description de la scène où Patrocle, fondant en pleurs, implore Achille de retourner au combat (XVI, 7-11), manifesterait aussi ces sentiments de protection et de tendresse. Achille lui-même compare son ami à une petite fille qui, courant vers sa mère, la supplie de la prendre dans ses bras. Dans les cas que nous venons d'évoquer, les trois guerriers secondaires sont assimilés à des petits enfants et les images employées nous mènent d'un monde hostile et plein de trahisons, celui de la guerre, à un environnement calme et sécurisé, celui créé par l'affection maternelle.

Des mots qu'Achille adresse à sa mère, Thétis, quand il lui demande son aide, on peut déduire que la maternité entraîne implicitement l'obligation d'aide permanente quel que soit l'âge ; ainsi, avant de lui demander son assistance, son fils lui rappelle : « $O$ mère, si tu m'as enfanté [...] » (Iliade, I, 355).

La tendresse qu'une mère manifeste pour ses enfants est aussi une constante qui transcende cette première étape et qui devient permanente (Slatkin 2011, p. 31-35). De cette même rencontre entre Achille et Thétis, nous reprenons l'extrait suivant qui décrit 
la réaction de la déesse : « [...] et sa mère auguste l'entend, du fond des abîmes marins [...] elle s'assied face à son fils en larmes, elle le flatte d'une main [...] Mon enfant, pleures-tu ? Quel deuil est venu à ton cœur? Parle, ne me cache pas ta pensée, que nous sachions tout tous les deux. » (Iliade, I, 365-367).

Les gestes de tendresse sont visibles dans le contact physique entre mère et enfants comme ceux décrits pour ne citer que quelques exemples, dans les rencontres entre Aphrodite et Énée (Iliade, V, 315), Dioné et Aphrodite (Iliade, V, 371) ou Hécube et Hector ( Iliade, VI, 252).

L'autre trait de caractère commun à toutes les mères et qui est aussi intemporel c'est leur capacité à convaincre leurs enfants ; l'idée n'est pas qu'elles parviennent toujours à cet objectif mais du moins que, si elles n'y arrivent pas, personne d'autre ne pourra le faire. Voilà ce qui pousse Zeus à demander à Thétis d'intercéder afin de convaincre Achille d'accepter les dons de Priam et de rendre le cadavre d'Hector (Iliade, XXIV, 75-140).

\section{La puberté et l'adolescence}

La demeure d'Ithaque dans laquelle, désespérés et impatients, Pénélope et Télémaque attendent le retour d'Ulysse qui leur manque (Reboreda Morillo 2006), se dresse comme un décor privilégié pour l'étude de la relation établie entre une mère et son fils pendant cette étape de croissance. À cet effet nous prenons comme point de départ les travaux de Nancy Felson-Rubin (1994, p. 67-91) et de Richard Heitman (2005, p. 50-62) que nous approfondissons et développons.

21 Vingt ans se sont écoulés depuis qu'Ulysse, basileus d'Ithaque, est parti en compagnie des guerriers aristocrates pour lutter dans la guerre de Troie. Ils ne sont jamais rentrés. Des nouvelles sur leur sort sont maintenant pratiquement inexistantes et, même si leur mort au combat n'a pas été constatée, l'hypothèse de leur décès commence à s'imposer. Dans l' oikos du héros, l'incertitude est palpable et ses proches sont continuellement écartés. Ces deux facteurs vont prendre de plus en plus d'importance quand une centaine de jeunes prétendants désirant se marier avec Pénélope s'installent pratiquement dans son foyer, transformant la vie en un banquet continu avec des manques de respect permanents visà-vis des propriétaires et de leurs hôtes (Leduc 2001, p. 285).

Centrons-nous d'abord sur Télémaque. Même si, en raison de son âge, Télémaque devrait pouvoir prendre le contrôle de la situation, il se montre incapable d'assumer la direction de l'oikos qui un jour sera à lui. De fait, sauf pour certains aspects ponctuels comme accueillir les étrangers, son attitude oscille entre les lamentations pour l'absence d'un père qu'il n'a jamais connu et la crainte de voir son domaine disparaître entre les mains d'insatiables prétendants.

Pour sa part, Pénélope, consumée par la douleur, une fois découverte la célèbre ruse de défaire la nuit ce qu'elle avait tissé le jour, se débat entre l'attente du retour d'Ulysse et le choix d'un nouvel époux (Berguen 1995, p. 210), avant même l'âge adulte de Télémaque. Comment se structure la relation entre la mère et le fils au milieu de ce chaos?

La première allusion à leur parenté est pour le moins singulière. La déesse Athéna, déguisée en Mentès, ami d'Ulysse, est accueillie comme hôte par Télémaque. Quand la déesse l'interroge sur son identité, Télémaque se définit de la façon suivante : «Que je 
sois bien son fils ? [...] ma mère me le dit: moi, je n'en sais pas plus ; à quel signe un enfant reconnaît-il son père? ? (Odyssée, I, 215-220)4.

Dans sa réponse, la déesse place elle aussi la lignée maternelle au-dessus de la paternelle : « Ne crois pas que les dieux aient refusé leur signe à cette descendance, quand c'est un pareil fils qu'enfanta Pénélope » (Odyssée, I, 222-224).

D'après Létoublon (2010), «il n'est visiblement pas absolument sûr d'être le fils d'Ulysse ». De fait, l'auteur affirme que le voyage de Télémaque à Pylos et à Sparte a comme principal but d'éclaircir son problème d'identité.

Ma réflexion sur ces affirmations se justifie en partie par cette image sociale de la femme un peu plus positive, déjà commentée, et, d'autre part, par l'idée qui renvoie à l'immaturité de Télémaque ; cette immaturité chez les jeunes gens était apparentée à la première étape de leur vie qui se déroulait dans un milieu féminin (Renaud et Wathelet 2002, p. 277). Comme le soulignait Moses Finley (1980, p. 90), il est évident que dans la société homérique on attendait d'un individu de vingt ans, d'une telle lignée et d'une telle classe, qu'il se développe plus vite et mieux, et qu'il montre la même capacité de réaction face aux événements qu'un adulte. Télémaque aurait dû passer à la phase éducative suivante puisqu'il lui manquait le référent masculin et pourtant il tarde à franchir ce pas. Cette singularité marque toute la première partie de l'œuvre même si cela n'empêche pas que Télémaque manifeste souvent une grande admiration pour Ulysse et un désir permanent de le voir revenir.

Cette absence de maturité a un résultat bénéfique pour Pénélope car elle peut ainsi justifier son indécision quant aux instructions d'Ulysse. Le héros, avant de partir, l'avait chargée de prendre soin de son domaine, de ses parents et de son fils, jusqu'à ce que " plus tard, quand tu verras de la barbe à ton fils, épouse qui te plaît et quitte la maison » (Odyssée, XVIII, 265-270); à ce moment-là elle devait choisir un nouveau mari et abandonner la maison. Il ne s'agissait pas simplement de constater un changement physique mais l'aptitude à gouverner.

Dans les circonstances décrites, seule Pénélope pouvait encourager cette maturité mais elle a choisi « d'arrêter » le temps (Reboreda Morillo 2010, p. 194-200), non seulement en tissant de jour et en défaisant de nuit (Berguen 1995, p. 212) le linceul de son beau-père Laërte, mais aussi en favorisant le fait que Télémaque demeure dans cette phase antérieure à l'âge adulte.

31 Seule l'intervention d'Athéna provoquera des changements dans l'oikos du héros. Un de ses premiers objectifs sera justement d'accélérer cette tardive maturité de Télémaque en remplaçant Pénélope dans sa fonction. Ce n'est qu'une fois adulte que Télémaque pourra aider son père dans le combat contre les nombreux prétendants. La déesse va lui suggérer deux idées : convoquer l'assemblée pour exposer aux aristocrates d'Ithaque les difficultés que traverse son foyer et voyager à Pylos et à Sparte afin d'y recueillir des nouvelles de son père. Si sa mort était confirmée, il devrait célébrer les rites funéraires exigés, donner sa mère à un autre époux et prendre en charge son héritage (Odyssée, II, 270). La mise à exécution de ces deux conseils sera la première immersion de Télémaque dans la sphère masculine et les conséquences seront immédiates et bientôt visibles pour les prétendants et pour sa mère quand il lui adresse ces mots :

Tu refuses, ma mère, à l'aède fidèle le droit de nous charmer au gré de son esprit?

[...] Prend donc sur tes pensées et ton cœur de l'entendre. Ulysse, tu le sais, ne fut pas seul à perdre la journée du retour ; en Troade, combien d'autres ont succombé ! Va! Rentre à la maison et reprends tes travaux, ta toile, ta quenouille; ordonne à 
tes servantes de se remettre à l'œuvre; le discours c'est à nous, les hommes, qu'il

revient, mais à moi tout d'abord, que je suis maître céans (Odyssée, I, 346-359). Nancy Felson-Rubin (1994, p. 71) associe cette réaction à la brusquerie de l'adolescence ; mais nous pouvons aller plus loin si nous déterminons que, pour la première fois, Télémaque montre une maturité en accord avec sa capacité à gouverner la maison. La réaction de Pénélope confirme cette idée : « Pénélope, étonnée, rentra dans sa maison; le cœur rempli des mots si sages de son fils; et lorsqu'à son étage, elle fut remontée avec ses chambrières elle pleurait encore Ulysse, son époux " (Odyssée, I, 360-364). Elle semble accepter cette nouvelle relation de subordination à son fils en raison de l'âge du garçon.

Antinoos, un prétendant qui a certaines possibilités de succès, associe lui aussi cette maturité naissante à un changement peut-être favorable et incite Télémaque à ordonner à sa mère de choisir un nouvel époux. La réponse de Télémaque souligne l'étroite relation que le jeune continue à maintenir avec sa mère en affirmant, entre autres, qu'il ne pense pas mettre à la porte la personne qui lui avait donné la vie et l'avait nourri (Odyssée, II, 130-132). Le poème souligne ainsi un attachement envers celle qui l'a enfanté, élevé et nourri ainsi qu'une nécessaire redevabilité.

Télémaque, après avoir convoqué l'assemblée, met le cap sur Pylos et Sparte : un voyage à vocation plus qu'initiatique et qu'il souhaite explicitement cacher à sa mère. Pénélope apprend en même temps son départ et l'embuscade que les prétendants, maintenant méfiants, trament contre son fils. Sa réaction est une autre preuve de la profondeur de son amour filial : «Et la reine, genoux et cœur brisés restait là sans pouvoir proférer un seul mot : ses yeux s'étaient emplis de larmes et sa voix si claire défaillait... » (Odyssée, IV, 702-705).

D'après Jean-Michel Renaud et Paul Wathelet (2002, p. 278), ce voyage "est présenté comme une descente symbolique aux Enfers » à l'issue de laquelle, Télémaque aura acquis toute une série de qualités extraordinaires indispensables pour pouvoir combattre à côté de son père. Les auteurs, qui établissent un parallélisme évident entre Ulysse et son fils, estiment que le voyage de Télémaque constitue une initiation de deuxième degré. La première initiation, celle qui marque le passage à l'âge adulte, a lieu avant la convocation de l'assemblée, au moment où Télémaque comprend qu'Athéna est à ses côtés (Iliade, I, 323).

Cependant Pénélope nie la capacité de décision de Télémaque affirmant même que si elle avait su à temps que celui-ci voulait entreprendre le voyage elle l'aurait retenu (Odyssée, IV, 729-734). Sans aucun doute Pénélope refusait encore de voir son fils se transformer en une personne adulte.

37 Face à cette attitude immobiliste, Athéna poursuit son objectif d'accélérer cette maturité pour cela, elle considère indispensable la rupture de ce lien maternel. Cette nécessité oblige peut-être la déesse à travestir la réalité en faisant l'affirmation suivante :

Car voici que son père et ses frères la pressent d'épouser Eurymaque [...] prends garde à ton insu, si quelqu'un de tes biens sortait de ton logis! Tu sais le cœur des femmes : c'est toujours la maison de leur nouveau mari qu'elles veulent servir; leurs fils d'un premier lit, l'époux de leur jeunesse ne comptent plus pour elles; il est mort, c'est l'oubli ! (Odyssée, XV, 16-24).

Dans cette même optique, Télémaque lui-même, chez Eumée et sans savoir que le mendiant qui était devant lui est son père, manifeste ouvertement des doutes sur l'intégrité des intentions de Pénélope, mais il exprime en même temps son respect en chargeant un serviteur d'aller prévenir sa mère de son heureux retour. 
39 Le coup de pouce définitif pour devenir adulte vient de la présence de son père et de la planification entre eux de la vengeance souhaitée; il rompt, à ses côtés, définitivement avec l'univers féminin, ce qui se perçoit dans ses paroles à Euryclée :

Nourrice, qu'a-t-on fait pour bien traiter notre hôte? Car je connais ma mère ! Et cette âme si sage est parfois étonnante pour tirer du commun des mortels la canaille et, sans égards, chasser les plus honnêtes gens (Odyssée, XVII, 129-133).

Malgré ces circonstances, l'affection maternelle sera une constante perceptible dans ces retrouvailles :

Elle prit dans ses bras son enfant et, pleurant, le baisant sur le front et sur ses deux beaux yeux, et laisse à travers des sanglots échapper ces mots rapides : Te voilà, Télémaque! ô ma douce lumière! J'ai cru ne jamais te revoir quand j'ai su qu'embarqué en secret, contre ma volonté, tu partais pour Pylos t'informer de ton père. Allons! Dis-moi, qu'as-tu rencontré ? Qu'as-tu vu ? (Odyssée, XVII, 38-44).

Néanmoins, Pénélope, consciente du changement opéré chez son fils, n'est pas disposée à assumer les avantages de cette maturité :

Télémaque, es-tu donc sans esprit et sans cœur? Tout petit, tes desseins étaient mieux réfléchis; te voilà grand; tu vas entrer dans l'âge d'homme : à te voir bel et grand, il n'est pas d'étranger qui ne te proclamât le fils d'un homme heureux ; mais parfois tu parais sans esprit et sans cœur (Odyssée, XVIII, 215-220).

42 C'est à ce moment qu'elle se souvient, en se lamentant, du conseil que son mari lui avait donné avant de partir pour Troie et qu'elle demande aux prétendants les cadeaux obligatoires avant son choix imminent. Marquant des différences avec l'univers masculin, Télémaque et Ulysse cachent consciemment à Pénélope la présence du héros et leurs plans pour le dénouement.

Lorsque tout est fini et qu'Euryclée lui annonce que son époux et son fils ont tué les prétendants, la première réaction de Pénélope est d'incrédulité, attitude que lui reproche son fils, selon le schéma indiqué :

Ton cœur est trop cruel, mère ! ô méchante mère ! De mon père, pourquoi t'écarter de la sorte ? [...] Auprès de lui, pourquoi ne vas-tu t'asseoir, lui parler, t'enquérir? [...] Fut-il jamais un cœur de femme aussi fermé ? [...] S'éloigner d'un époux quand après vingt années de longs maux et d'épreuves, il revient au pays ! [...] Ah! Ton cœur est toujours plus dur que la roche (Odyssée, XXIII, 97-103).

On peut conclure que finalement, mère et fils se font le même type de reproches : l'absence de cœur!

\section{La maturité et la guerre}

Comme je l'ai décrit au début de mon exposé, l'activité belliqueuse était la principale occupation des aristoi. En conséquence, les plus grandes craintes d'une mère quand son fils atteignait la maturité étaient liées aux suites néfastes qui découlaient de la guerre ; la plus significative et la plus récurrente étant la mort, pouvant survenir loin de la maison, en terrain ennemi, ou à proximité de la communauté, comme il était le cas de Troie. Ces mots sont ceux prononcés par la déesse Thétis, dans ce cas pleinement consciente du destin de son fils mortel, et que nous pourrions prêter à n'importe quelle mère des presque éternels guerriers grecs (Slatkin 2011, p. 42-71).

Ah! Misérable que je suis! Mère infortunée d'un preux! J'ai donné la vie à un fils, un fils puissant et sans reproche, le plus grand des héros; il a grandi pareil à une jeune pousse, et, après l'avoir nourri, comme un plant au flanc du vignoble, je l'ai 
envoyé, sur des nefs recourbées, au pays d'Ilion, se battre contre les Troyens. Et je ne dois plus le revoir ni l'accueillir rentrant chez lui (Iliade, XVIII, 54-60). de Thétis quand elle associe les difficultés de mettre au monde et d'élever un enfant aux rares compensations obtenues par la mère d'un guerrier, c'est-à-dire d'un homme : vivre dans la perpétuelle angoisse pour le sort de son fils qui s'achèvera probablement par la mort. De cela, Nicole Loraux (1990), propose une magnifique analyse, plus centrée sur l'époque classique.

Cependant, dans les poèmes homériques, quand des affirmations générales sur les conséquences que la mort d'un guerrier aura sur des tiers sont faites, les mères ne sont jamais au premier plan; cette place est occupée par les épouses et les fils mineurs car on sous-entendait que les femmes, une fois mariées, dépendaient de la protection de leur mari. Les allusions sont nombreuses dans l'Iliade et confirment cette réalité ; le cas le plus éclairant est celui d'Andromaque et d'Astyanax, respectivement femme et fils d'Hector (Le Meur-Weissman 2009); comme le dit Andromaque :

Pauvre fou! Ta fougue te perdra. Et n'as-tu pas pitié non plus de ton fils si petit, ni de moi, misérable, qui de toi bientôt serai veuve ? (Iliade, VI, 407-410).

\section{éloquente de son amour filial :}

Elle disait et moi, à force d'y penser, je n'avais qu'un désir : serrer entre mes bras l'ombre de feu ma mère [...] Trois fois, je m'élançai ; tout mon cœur la voulait. Trois fois, entre mes mains, ce ne fut plus qu'une ombre ou qu'un songe envolé. L'angoisse me poignait plus avant dans le cœur. Je lui dis, élevant la voix, ces mots ailés : Mère, pourquoi me fuir, lorsque je veux te prendre? Que, du moins chez Hadès, nous tenant embrassés, nous goûtions, à nous deux, le frisson de sanglots ! [...] La noble Perséphone en suscitant ton ombre, n'a-t-elle donc voulu que redoubler ma peine et mes gémissements? (Odyssée, XI, 204-214).

51 Toutefois, la situation la plus habituelle décrite dans les poèmes homériques est, à l'inverse, celle où les fils, combattants aguerris, meurent sur le champ de bataille et, si les circonstances permettent de récupérer le cadavre et de le ramener à la maison, sont enterrés par des êtres chers selon les rites funéraires exigés. Il est évident qu'au-delà de la crainte de souffrir pour la mort d'un fils, l'incertitude s'étend à la possibilité de lui offrir des funérailles en accord avec les coutumes religieuses.

52 Le rôle central que les femmes de la famille, mères et épouses, détenaient dans les rites funèbres jusqu'au $\mathrm{VI}^{\mathrm{e}}$ av. J.-C., est une autre preuve que l'on tenait les femmes en une plus haute considération qu'à l'époque classique. 

membres de sa famille. Curieusement, même s'il était de mauvais augure de pleurer quelqu'un encore en vie (Alexiou 2002, p. 4), bien que les probabilités de la mort du héros soient élevées, dans le chant VI de l'Iliade, Andromaque est décrite gémissante, son fils dans les bras, pleurant en compagnie de ses servantes, alors que son époux est encore vivant (v. 370-375 et v. 497-503). Elle même déclare à Hector : «malheureuse qui serai veuve bientôt car bientôt les Achéens t'immoleront, en réunissant tous leurs efforts " (v. 408-409). 
60 Hécube, sa mère, se montre moins bouleversée quand son fils arrive au palais. Après lui avoir pris la main dans un geste caractéristique de tendresse entre mère et fils, elle lui offre du vin revigorant et se dit prête à collaborer, avec des offrandes à Athéna, pour que celle-ci se montre favorable aux guerriers troyens (Iliade, VI, 252-310). Comme les autres mères « épiques ", elle tente de défendre et de protéger son fils.

61 L'intervention suivante d'Hécube sera tout le contraire de la tranquillité antérieure ; elle a lieu juste avant la mort d'Hector, quand elle aperçoit Achille. Avec les mots déchirants déjà cités pour l'allaitement, elle supplie son fils de ne pas affronter l'Achéen (Iliade, XXII, 79-90). L'analyse pertinente de Nicole Loraux (1990, p. 58-59) montre comment, face au dénouement imminent, la mère devient folle, dépossédée avant l'heure de son fils, et souligne sa crainte de voir son cadavre outragé et de ne pas pouvoir lui offrir les rites funèbres. En ce moment de désespoir, elle évoque, comme nous l'avons déjà vu, le bonheur d'avoir senti leurs corps proches pendant l'allaitement (Iliade, XXII, 82-85).

Quand Hécube observe depuis les murs de la citadelle comment Achille tue son fils, elle commence les rites correspondant aux lamentations funèbres: elle jette son voile, s'arrache les cheveux et lance un cri de douleur (XXII, 79-90); ces actions sont le signe que les autres êtres chers peuvent commencer leurs lamentations. Une fois la mort avérée, la deuxième incertitude est confirmée à la vue des outrages qu'Achille et les Achéens font subir à ce corps qui n'était pas prédestiné à ces rites funéraires qui permettaient un digne adieu à l'être cher pour que sa psuchê suive son chemin naturel vers le domaine d'Hadès.

63 Malgré le manque d'espoir, Priam, faisant fi des suppliques de son épouse résignée, se dirige vers le camp des Grecs afin d'offrir à Achille une appréciable rançon pour récupérer le corps de son fils. Nous avons déjà dit combien l'intercession de Thétis sera fondamentale.

64 L'Iliade se termine avec les rites que les femmes les plus proches accomplissent pendant la cérémonie funèbre. La femme et la mère d'Hector "s'arrachent les cheveux, elles se jettent sur les chariots aux bonnes roues, elles touchent la tête du mort ». Pendant l' ekphora, deux groupes parfaitement différenciés vont intervenir dans les lamentations : d'abord, les chanteurs professionnels qui entonnent le thrênos et en réponse, les femmes les plus proches exprimant leur plainte, le goôs.

La première, Andromaque, qui s'adresse directement à son mari en lui décrivant l'avenir funeste qui les attend son fils et elle à cause de sa disparition; ensuite, Hécube qui détaille à son fils sa profonde tendresse et sa douleur consécutive à sa mort: durant ses lamentations, on observe surtout sa résignation quand elle fait référence, de façon générique, aux autres fils qu'Achille a conduits vers l'esclavage. Finalement, Hélène fait l'éloge de l'excellent comportement que son beau-frère a toujours eu envers elle, bien qu'elle fût la cause de tous les maux.

On peut constater, pour l'ensemble des interventions, que les éloges ne sont pas abondants et quand ils font leur apparition, aucun ne souligne les qualités de guerrier d'Hector; on n'aperçoit pas non plus de vision positive ou de fierté pour la mort au champ de bataille. Les lamentations transmettent la douleur de l'absence, la rupture des liens familiaux - entre époux, entre mère et fils - la douleur pour la vie future et le regret individualisé du défunt que ce soit pour ses qualités physiques ou pour sa personnalité. 


\section{Conclusions} maternelle s'affichait par le biais de leur pouvoir de conviction. Il est aussi récurrent que les fils demandent l'aide de leur mère et que celles-ci y accèdent dans la mesure de leurs possibilités. pouvoir célébrer les rites funèbres en leur honneur. rituel privé en une affaire publique et d'État. 


\section{BIBLIOGRAPHIE}

Alexiou M. (2002), The Ritual Lament in Greek Tradition, Boston.

Bile M. et Klein J. (2008), « Hector et les principaux personnages féminins de l'Iliade. Études des scholies ", Gaia 11, p. 121-127.

Bergen A. (1995), « The (Re)Marriage of Penelope and Odysseus: Architecture, Gender, Philosophy. A Homeric Dialogue », dans Carter B. et Morris S. éd., The Ages of Homer. A Tribute to Emily Townsend Vermeule, Austin.

Bruit Zaidman L. (2001) [1992 en français], « Las hijas de Pandora. Mujeres y rituales en las ciudades », dans Duby G. et Perrot M. éd., Historia de las mujeres. 1. La Antigüedad, Madrid, p. 394-444.

De Nazaré Ferreira L. (2000), « A evocaçaô do mundo infantil na Ilíada », Humanitas LII, p. 53-76.

De Paco Serrano D. (2007), « Madres divinas y madres humanas en la Ilíada », dans Calderón Dorda E. et Morales Ortiz A. éd., La madre en la antigüedad: literatura, sociedad y religión, Madrid, p. 61-76.

Felson-Rubin N. (1994), Reading Penelope. From Character to Poetics, Princeton-New Jersey.

Finley M. I. (1980) [1961], El mundo de Odiseo, Mexico.

Heitman R. (2005), Taking her Seriously. Penelope and the Plot of Homer's Odyssey, Michigan.

Holst-Warhaft G. (1995), Dangerous Voices. Women's Laments and Greek Literature, LondresNew York.

Leduc C. (2001) [1992 en français], « ¿Cómo darla en matrimonio ? », dans Duby G. et Perrot M. éd., Historia de las mujeres. 1. La Antigüedad, Madrid, p. 271-336.

Le Meur-Weissman N. (2009), « Astyanax. Les enfants et la guerre dans l'Iliade », Gaia 2009, p. 29-43.

Létoublon F. (2010), « Télémaque est-il le fils d'Ulysse ?», Gaia 2010, p. 137-152.

Loraux N. (1981), L'Invention d'Athènes. Histoire de l'oraison funèbre dans la « cité classique ", ParisLa Haye.

- (1989), « Le lit, la guerre », L’Homme 21, 1981, p. 37-67 [repris dans Les expériences de Tirésias, Paris, 1989, p. 29-53].

- (1990), Les Mères en deuil, Paris.

Molas Font Mª . D.(2002), « Engendrar y parir en la Ilíada y en la Odisea », dans Molas Font Mª. D. éd., Vivir en femenino. Estudios de mujeres en la antigüedad, Barcelone, p. 153-178.

Molinos Tejada Mํ. T. (2001), «Las nodrizas en la escena clásica », El Fil d'Ariadna 4, Bari, p. 299-312.

- (2005), « Madres y nodrizas en la Antigüedad », dans Pedregal A. et González M. éd, Venus sin espejo. Imágenes de mujeres en la Antigüedad clásica y el cristianismo primitivo, Oviedo, p. 55-80.

Mossé C. (1983), La femme dans la Grèce antique, Paris. 
Reboreda Morillo S. (2006), « Los reencuentros de Odiseo en Ítaca », biTARTE 40, p. 49-68.

- (2009), « Penélope: La maternidad en el caos », dans Cid López R. éd, Madres y maternidades. Construcciones culturales de la civilización clásica, p. 47-66.

- (2010), «El nostos de Odiseo desde la perspectiva de los que esperan: La activa inactividad de Penélope ", dans Alvar Nuño A. éd., El viaje y sus riesgos. Los peligros de viajar en el mundo grecoromano, p. 185-294.

Renaud J.-M. et Wathelet P. (2002), «L'initiation de Télémaque dans l'Odyssée », dans Hurst A. et Létoublon F. éd., La Mythologie et l'Odyssée. Hommage à Gabriel Germain. Actes du colloque international de Grenoble, 20-22 mai 1999, Genève, p. 273-286.

Scheid-Tissinier É. (1999), L'Homme grec aux origines de la cité (900-700 av. J.-C.), Paris.

Slatkin L.M. (2011), The Power of Thetis and Selected Essays, Cambridge-Londres.

\section{NOTES}

1. Ce travail s'intègre aussi dans le projet de recherche intitulé "Clés diachroniques de la divergence sociale entre les constructions symboliques et les constructions juridiques de la maternité » dirigé par le professeur Rosa María Cid López et financé par le Ministère de l'Éducation, de la Culture et du Sport du gouvernement espagnol (HAR 2009-10035 Hist.).

2. Cf. Iliade, XVI, 188-189. Pour les textes de l'Iliade, j'ai toujours utilisé la traduction de Paul Mazon, éditée par les Belles Lettres, Paris. Voir Loraux 1989.

3. En plus de l'exemple d'Hécube voir celui de Thétis avec Achille : Iliade, I, 415.

4. Pour les textes de l'Odyssée, j'ai toujours utilisé la traduction de Victor Bérard, éditée par les Belles Lettres, Paris.

\section{RÉSUMÉS}

Cette recherche est axée sur l'idée que les textes homériques transmettent des bribes d'informations sur la conception de la maternité. Je suis bien consciente des difficultés d'une telle tâche, en commençant par la célèbre question homérique. Ce que je me propose c'est de recueillir tous les indices de la relation mère/fils/fille en suivant, dans la mesure du possible, l'ordre des étapes de la vie, depuis la plus tendre enfance jusqu'à la maturité ; ce choix m'obligera à aborder l'Iliade ou l'Odyssée en fonction du sujet considéré. Les analyses incluront aussi bien des références au monde humain qu'au monde divin; cette liberté est admissible parce que je me concentrerai toujours sur des enfants humains dont les mères sont soit humaines soit divines. Les sentiments des unes et des autres peuvent être comparables car leurs enfants sont tous mortels.

This investigation centres on the idea that the Homeric texts transmit some pieces of information on the greek conception of maternity. I am conscious that my task presents many difficulties, beginning for the famous "Homeric question". My proposal is to collect all the evidence of the relation mother/son/daughter following, as far as possible, the order of the 
stages of growth, from the most tender infancy up to the maturity; this choice will force me to approach the Iliade or the Odyssey depending on the subject that I will tackle. The analyses will include also mentions to both the human and the divine world; this liberty is possible because I always focus on human children, sometimes of human mothers, sometimes of divine mothers. The feelings of some and others can be comparable because they share the fact that their children are mortal.

INDEX

Mots-clés : maternité, accouchement, allaitement, fils, fille, protection, tendresse

Keywords : maternity, labour, breast-feed, sons, daughter, tenderness

\section{AUTEUR}

\section{SUSANA REBOREDA MORILLO}

Université de Vigo, Espagne 